\title{
Molecular characterization of Lingual antimicrobial peptide in the female reproductive tract of Buffalo
}

\author{
Dhanya J oseph* and Tukaram More \\ Department of Animal Biochemistry, \\ Indian Veterinary Research Institute, I zatnagar, 243 122, India. \\ Corresponding author email: jdhanya7@gmail.com
}

\begin{abstract}
Bubalus bubalis (Ruminantia: Bovidae, Bovinae) is an economically important animal of many Asian countries, making significant contribution to milk and meat production. Sub clinical infection of the reproductive tract is one of the important causes for reduced reproductive efficiency in dairy herd of buffaloes. Antimicrobial peptides are component of innate immune system which helps in augmenting the resistance to infection at epithelial surfaces e.g reproductive tract epithelium. In this study we have identified a ß-defensin called Lingual Antimicrobial Peptide (LAP) in buffalo reproductive tract. Interestingly the gene was $100 \%$ identical to the LAP isolated from the tongue epithelium of Bos taurus. The $195 \mathrm{bp}$ cDNA of LAP codes for 64 amino acids and of which $50 \%$ are cationic amino acids. Phylogenetic studies indicate that LAP of reproductive epithelium of buffalo is different from other beta defensins isolated from the various tissues of same species, but all beta defensin were found to have the same progenitor gene. It is concluded that buffalo reproductive tract epithelium lining contains LAP.
\end{abstract}

Keywords: Antimicrobial Peptide, Reproduction, Buffalo, Milk Production, Meat Production, Amino acid.

\section{I ntroduction}

Antimicrobial peptides are important constituents of the innate immune defense of all life forms including mammals, amphibians, insects and plants. Lingual antimicrobial peptide (LAP) was first isolated from the tongue epithelium of bovine (Schonwetter et al., 1995). Subsequent investigation revealed the expression of LAP in a wide variety of tissues like trachea, intestine, mammary gland, female reproductive tract of bovine and also in buffalo tongue and mammary gland. LAP has broad spectrum antibacterial activity, antifungal activity and immunomodulatory activity which is important in limiting the establishment of infection on epithelial surfaces (Stolzenberg et al., 1997). The present study reports the expression pattern of LAP along the female reproductive tract, its cDNA sequence, the deduced amino acid sequence, degree of identity of the LAP with other defensin gene and its phylogenetic relation.

\section{Material and Methods}

The female reproductive tract sample of a buffalo was collected from local abattoir within 30 minutes after their slaughter. Six female reproductive tracts were used for the study. The clinical history of the animal was unknown. The animals were 5-6 years of age. The reproductive organ was washed thoroughly with $1 \mathrm{X}$ phosphate buffered saline of $\mathrm{pH} 7.4$ to remove blood and other cells adhering to the epithelium.

Total RNA was isolated separately from $200 \mathrm{mg}$ of epithelial scrapings lining the lumen of vagina, cervix, uterus and oviduct using 'Total RNA isolation system' (Promega, USA). The integrity of the RNA was confirmed by $1 \%$ agarose gel electrophoresis. The purity of the RNA was checked by spectrophotometer, measuring the A260/A280. Qiagen (Germany) RTPCR Kit was used to produce cDNA from the total RNA template, following the manufactures protocol. Complete cDNA sequence of LAP including start and stop codon was obtained by RT-PCR using the following primers: forward 5'-CGG CAC CGA CAG CAT GAG G-3' and reverse 5'-GCC ACG TCT TCG CCT TCT TT-3. Both primers were designed based on bovine LAP sequence published in NCBI (Accession No: S76279). The annealing temperature was standardized (Mastercycler, Eppendrof, Germany) and the annealing temperature of 560C for 1 minute was found to be optimum. For RT-PCR the initial denaturation was carried out at $950 \mathrm{C}$ for 15 minutes, followed by 30 cycle of amplification with denaturation at $940 \mathrm{C}$ for 1 minute, primer annealing at $560 \mathrm{C}$ for 1 minute and elongation at $720 \mathrm{C}$ for 45 second. The final extension was done at $720 \mathrm{C}$ for 10 minutes. The concentration of RNA in the reaction 
Figure-1: The cDNA sequence of buffalo reproductive tract LAP along with deduced peptide sequence.

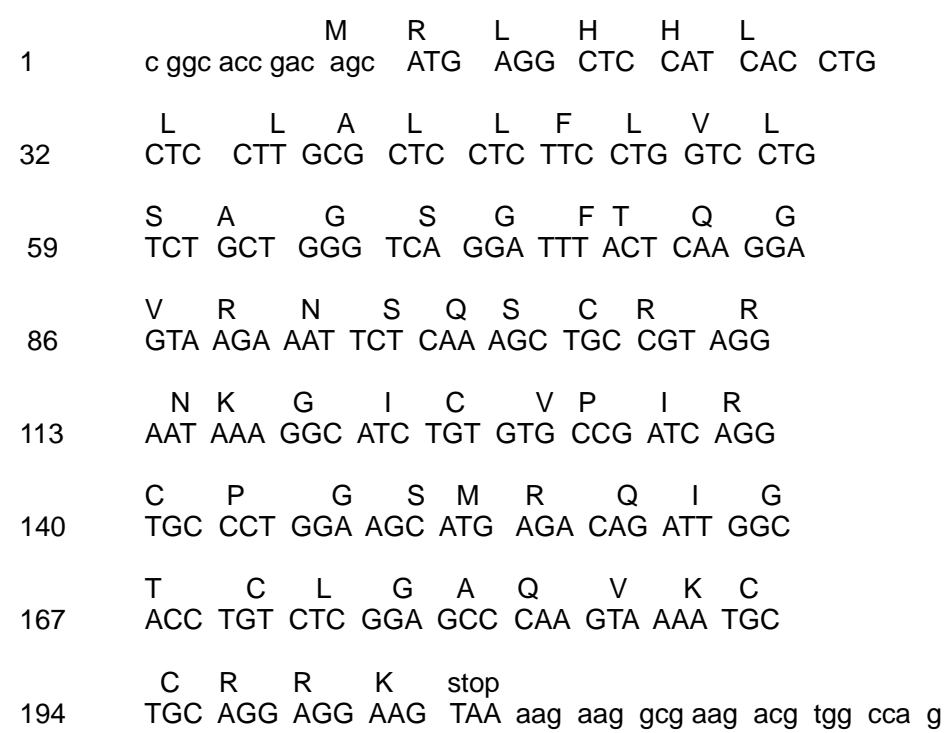

mixtures were $20 \mathrm{ng}$. A negative control (no template) was also included to test for non specific amplification.

A single band of RT-PCR product was visualized after electrophoresis using $1.5 \%$ agarose gel with ethidium bromide and the size of the product was confirmed using Gene ruler, Fermentas. The RT-PCR product was further cloned into the pTZ57RT-cloning vector (Ins T/A clone TM PCR product cloning Kit, Fermantas). The positive clones were selected based on blue and white screening and confirmed by plasmid PCR. Four positive clones each from vagina, cervix, uterus and oviduct were stab cultured in LB agar containing ampicillin $(100 \mathrm{mg} / \mathrm{ml})$ and sent for DNA sequencing to University of Delhi, South Campus, New Delhi. The gene was sequenced by Sanger's dideoxy method in an automated DNA sequencer. To analyze the cDNA sequence, LAP gene expressed in the reproductive tract was aligned with other reported sequence of B-defensin from different species using clustal method of 'Meg Align' programme of Laser gene software (DNA star Inc; USA). The sequences used for comparison were collected from NCBI website.

\section{Results and Discussion}

On RT-PCR of RNA isolated from vagina, cervix, uterus and oviduct using LAP primers we were able to visualize a single $230 \mathrm{bp}$ product in each lane by running a $1.5 \%$ agarose gel stained with ethidium bromide. The total RNA collected from all the six samples was positive for the 230bp cDNA sequence. The DNA sequence of the cloned LAP gene and the deduced amino acid is shown in Fig.1.The gene sequence was submitted to Genbank and was given the accession No: EF 630358. The open reading frame (ORF) of buffalo LAPcDNA sequence showed $100 \%$, $87.2 \%$ and $100 \%$ similarity with Bos taurus LAP, tracheal antimicrobial peptide (TAP) and Bubalus bubalis udder LAP respectively. The degree of similarity and phylogenetic relationship of the reproductive tract LAP in comparison to other Bdefensin and LAP sequences reported in NCBI is shown in Fig. 2 and Fig. 3 respectively. In the present study we have found the expression of LAP in vaginal, cervix, uterine and oviduct epithelium based on its nucleotide sequence.

The ORF of the gene was 195bp.The ORF extends from the 15th adenine base to 208th adenine base. Russell et al.,(1996) reported increased expression of LAP mRNA in mucosal surfaces exposed to bacterial lipopolyssacride and tumor necrosis factor alpha. When the nucleotide sequence of reproductive tract LAP was compared to the lingual LAP sequence from Bos taurus, $100 \%$ similarity was noticed in the ORF, while $87.2 \%$ similarity was reported with Goat LAP sequence. This high degree of similarity demonstrate remarkable inter species conservation of this gene.

The high level of homology in Bubalus bubalis, Bos taurus and Ovis aries also predicts that the functions of these peptides are important in disease 
Figure-2: Percent divergence of nucleotide sequence of buffalo reproductive tract LAP with LAP and other beta defensin gene from different species. NCBI accession number of the gene is also mentioned for each species.

\begin{tabular}{|c|c|c|c|c|c|c|c|c|c|c|c|c|c|c|c|c|c|c|c|}
\hline & 1 & 2 & 3 & 4 & 5 & 6 & 7 & 8 & 9 & 10 & 11 & 12 & 13 & 14 & 15 & 16 & 17 & & \\
\hline 2 & 0.0 & --- & 87.5 & 95.9 & 100.0 & 87.2 & 85.9 & 87.2 & 87.7 & 87.2 & 88.7 & 33.3 & 47.9 & 52.8 & 35.4 & 52.8 & 33.8 & 2 & EF418028 Bubalus bubalis LAP \\
\hline 3 & 12.7 & 12.7 & --- & 87.5 & 87.5 & 80.7 & 85.4 & 85.4 & 85.9 & 85.4 & 82.3 & 30.2 & 52.1 & 52.6 & 38.0 & 52.1 & 29.2 & 3 & AY392452 Bubalus bubalis BNDB-4 \\
\hline 5 & 0.0 & 0.0 & 13.2 & 4.2 & --- & 87.2 & 85.9 & 87.2 & 87.7 & 86.3 & 88.7 & 33.0 & 47.9 & 52.8 & 35.4 & 52.8 & 33.5 & 5 & S76279 Bos taurus LAP \\
\hline 6 & 13.5 & 13.5 & 20.2 & 11.0 & 13.5 & --- & 76.6 & 82.6 & 83.1 & 85.6 & 86.2 & 29.7 & 48.4 & 55.4 & 35.9 & 57.4 & 33.3 & 6 & NM174776 Bos taurus TAP \\
\hline 7 & 14.0 & 14.0 & 14.3 & 13.9 & 14.4 & 23.4 & --- & 80.2 & 80.7 & 78.6 & 79.2 & 32.3 & 52.6 & 48.4 & 33.9 & 48.4 & 30.7 & 7 & NM174775 Bos taurus BNBD-4 \\
\hline 8 & 12.9 & 12.9 & 13.9 & 11.1 & 12.9 & 17.9 & 18.5 & --- & 99.5 & 84.1 & 83.6 & 30.8 & 53.1 & 51.8 & 36.9 & 51.8 & 33.8 & 8 & NM175703 Bos taurus DEFB-1 \\
\hline 12 & 97.1 & 97.1 & 96.2 & 88.4 & 97.4 & 101.5 & 108.4 & 95.0 & 93.0 & 86.8 & 95.0 & --- & 27.6 & 28.7 & 31.8 & 29.2 & 63.3 & 12 & NM214442 Sus scrofa BD-2 \\
\hline 13 & 53.6 & 53.6 & 47.8 & 52.5 & 54.3 & 55.4 & 53.0 & 48.5 & 48.4 & 55.4 & 52.5 & 136.4 & --- & 50.0 & 29.2 & 51.0 & 31.3 & 13 & AF068861 Norway Rat BD-2 \\
\hline 14 & 56.8 & 56.8 & 56.4 & 54.5 & 57.1 & 52.7 & 62.4 & 56.8 & 55.7 & 57.6 & 55.5 & 116.9 & 52.2 & --- & 34.4 & 98.5 & 34.4 & 14 & AF 209855 Chimpanzee BD-2 \\
\hline 15 & 85.2 & 85.2 & 75.8 & 76.8 & 83.3 & 71.3 & 78.5 & 79.3 & 80.8 & 68.5 & 72.1 & 141.3 & 111.1 & 95.7 & --- & 36.4 & 25.6 & 15 & AF033336 Chicken prepro BD \\
\hline 16 & 56.8 & 56.8 & 57.6 & 54.5 & 57.1 & 50.4 & 62.4 & 56.8 & 55.7 & 57.6 & 55.5 & 126.0 & 52.2 & 1.6 & 90.9 & --- & 33.3 & 16 & AF288286 Rhesus monkey BD-2 \\
\hline 17 & 84.1 & 84.1 & 93.3 & 88.7 & 84.4 & 98.9 & 102.4 & 89.8 & 90.6 & 87.0 & 95.6 & 39.8 & 108.3 & 114.3 & 140.0 & 118.3 & --- & 17 & NM005218 Human DEFB1 \\
\hline & 1 & 2 & & 4 & & & 7 & 8 & & 10 & 11 & 12 & & 14 & 15 & 16 & 17 & & \\
\hline
\end{tabular}

resistance in these animals throughout the million years of independent evolution. Crovella (2005) reported substantial variation in antimicrobial peptides among mammalian species. Such variation was also noted with buffalo reproductive tract LAP sequence and other ?-defensins. For example, LAP has $88.7 \%$ sequence similarity with goat 3 -defensin-2, while only $52.8 \%$ with monkey beta defensin and $33.8 \%$ with human B-defensin (Fig.2).

The rapid co-evolution of host defense peptide, as well as of the corresponding microbial counter strategies, including resistance and virulence of these microbes might have helped to drive diversity in all biological kingdoms (Yount et al., 2006). On comparison of buffalo reproductive LAP with other Bdefensin nucleotide sequences reported from the same species, $95.9 \%$ similarity was seen with enteric $\beta$-Defensin (EBD) and $87.5 \%$ similarity was seen with Bovine Neutrophil B-Defensin 4 (BNBD4). These phylogenetic analysis data indicated that LAP, EBD and BNBD4 has evolved from a common ancestor Bdefensin gene (Fig.3). When BNBD4 and EBD gene

Figure-3. : Phylogenetic tree drawn comparing the nucleotide sequence of buffalo reproductive tract LAP with LAP and other beta defensin gene from different species

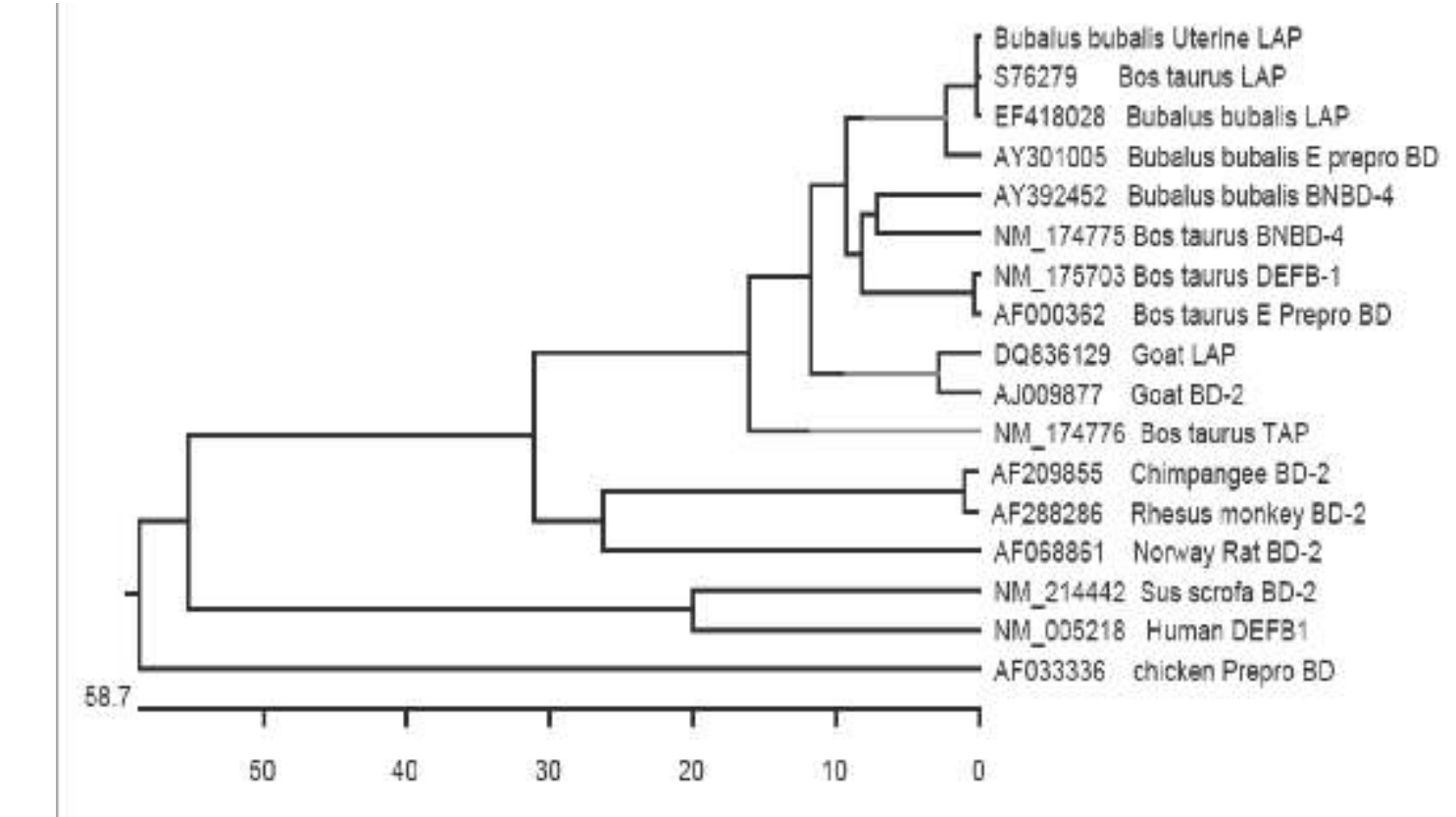


Figure-4: Agarose gel electrophoresis of amplified PCR product with ladder. Lane L: 100bp ladder. Lane 1, 2, 3, \&4: PCR product of buffalo vagina, cervix, uterus and oviduct LAP.

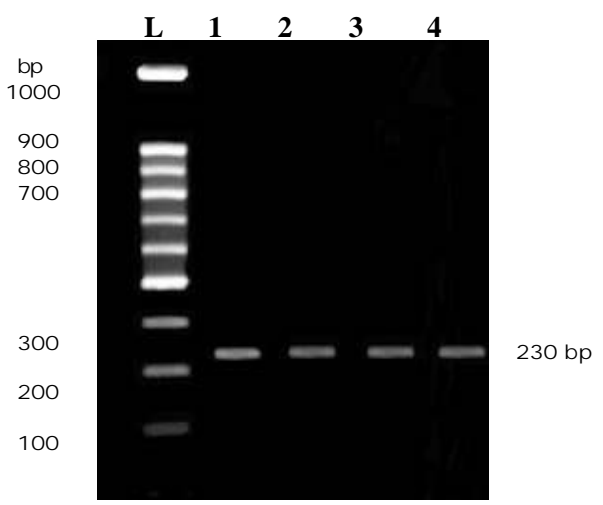

of Bos taurus was compared with buffalo LAP gene, $87 \%$ similarity was noted in nucleotide sequence (Fig.2).

The protein sequence predicted from the nucleotide sequence contained 65 amino acid residues including the six-cysteine residue, which is characteristic of B-defensin (Ganz and Leher, 1995). The prepropeptide obtained by conceptual translation of cDNA had a molecular mass of 7040.54 Da, with an isoelectric point of 11.324 and a net charge of 11.055 at $\mathrm{pH}$ 7. The initiation codon AUG is within the Kozak consensus sequence C GGC ACC GAC AGC AUG. The peptide has $50 \%$ of cationic residues like lysine, arginine and histidine while the other $50 \%$ is made of hydrophobic amino acid.

This finding is in accordance with the description of cationic antimicrobial peptides by Hancock and Diamond (1998) ; who described antimicrobial peptides as gene encoded prepropeptides that are activated by one or more proteolytic cleavage, short with 10-50 amino acid residues, have a net positive charge (+2 to +9$)$ and a substantial proportion of hydrophobic residues. The signal peptide was found to be 21 amino acid long as predicted by Signal P3.0 server using neural network and hidden Markov model (HMM) trained one eukaryotes (http:/www. ebs.dtu.dk/services/signalP.3/). The most likely site for cleavage to release the mature peptide is between position 21(phenylalanine) and 22(threonine), which is similar to Bos taurus LAP.

\section{Acknow ledgement}

Assistance by Dr.Hemen Das, Veterinary College, Anand campus in the form of valuable suggestion and interest is acknowledge gratefully. The authors are also thankful to Indian Council of Agricultural Research and Indian Veterinary Research Institute for facilities provided.

\section{References}

1 Crovella, S. (2005). Primate beta defensin-structure, function and evolution. Current Protein Peptide Science. 6:7-21.

2 Ganz, T and Lehrer, R.I. (1995). Defensins. Pharmacology and Therapeutics., 66:191-205.

3 Hanhock, R.E and Diamond, G. (1998).Cationic peptides: a new source of antibiotics. Trends in Biotechnology., 16:8288 .

4 Russell, J.P., Diamond, G., Traver, A.P., Scanlin, T.F and Brevins, C.L.(1996). Coordinate induction of two antibiotic genes in the tracheal epithelial cells exposed to the inflammatory mediatory lipopolyssachride and tumor necrosis factor alpha. Infection and Immunity., 64:15651568.

5 Schonwetter, B.S, Stolzenberg, E.D and Zasloff, M.A.(1995). Epithelial antibiotics induced at the site of inflammation. Science., 267:1645-1648.

6 Stolzenberg, E.D., Anderson, G.M., Ackermann, M.R., Whitlock, R and Zasloff, M.(1997).Epithelial antibiotic induced in states of diseases. Proceeding of National Academ of Science., 94:8686-90.

7 Yount, N.Y and Yeaman, M.R.(2006).Structural congruence among membrane active host defense polypeptide of diverse phylogeny. Biochim. Biophys. Acta. 9:1373-1386.

$$
* * * * * * * *
$$

